\title{
Role of Ubiquitin-Conjugating Enzyme UBE2C in Gastrointestinal Cancers
}

\author{
Ce Guo 1,3,4, Xing Guo², Zhen Wei³, Qian Wang3,4, Huiqing Zhang,3* \\ ${ }^{1}$ Chengde Medical University, Chengde 067000, Hebei Province, China \\ ${ }^{2}$ Oncology Department, the First Central Hospital of Baoding, Baoding 071000, Hebei Province, China \\ ${ }^{3}$ General Surgery, the First Central Hospital of Baoding, Baoding 071000, Hebei Province, China \\ ${ }^{4}$ Baoding Key Laboratory of Gastrointestinal Cancer, the First Central Hospital of Baoding, Baoding 071000, Hebei \\ Province, China \\ *Corresponding author: Huiqing Zhang, zhang13931386542@163.com

\begin{abstract}
Ubiquitin-conjugating enzyme UBE2C is one of the important members of ubiquitin-proteasome pathway (UPP). Amplification and/or overexpression of UBE2C have been reported in many malignancies, and a high expression of UBE2C is associated with poor clinical outcomes. In this review, the pathological role of dysregulated UBE2C in gastrointestinal cancers and its potential role as a diagnostic and/or a prognostic marker as well as a therapeutic target in these cancers are discussed.
\end{abstract}

Keywords: UBE2C; Digestive tract; Gastrointestinal cancers

Publication date: September 2021; Online publication: September 30, 2021

\section{Introduction}

Ubiquitination is an essential post-translational modification of proteins with diverse cellular functions. As a catalytic cascade, ubiquitination is accomplished sequentially, involving ubiquitin-activating enzymes (E1), ubiquitin-conjugating enzymes (E2), and ubiquitin ligases (E3) ${ }^{[1,2]}$. The human genome encodes two E1 enzymes, 38 E2 enzymes, and over 600 E3 enzymes, constituting a multiplex ubiquitination machinery to proceed the large variety of ubiquitin modifications ${ }^{[3-6]}$. Central in this catalytic cascade is the family of ubiquitin-conjugating enzymes (E2), which is characterized by the presence of a highly conserved ubiquitin-conjugating (UBC) domain ${ }^{[7]}$. By coupling activation of the ubiquitin molecule to downstream conjugation events, E2 determines the ubiquitination type of the downstream substrate, either with a single molecule or as a chain. Furthermore, while E3 is responsible for substrate specificity, E2 is the critical determinant for selection of the lysine site to construct ubiquitin chains, which thereby directly determines the cellular fate of the substrate ${ }^{[8]}$. E2 enzymes can be classified into four classes based on the existence of additional extensions to the catalytic UBC-fold. Class I E2s consist only of the UBC-fold, class II and class III E2s have either $\mathrm{N}$ - or C-terminal extensions, whereas class IV E2s have extensions at both terminals ${ }^{[9]}$. These extensions endow distinct E2s with important functions, such as subcellular localization, stabilization of the interaction with E1s, or modulation of activity of the interacting E3s.

UBE2C, belonging to class II E2 enzymes, is known to interact with the anaphase-promoting complex/cyclosome (APC/C) E3 ligase to promote cell cycle through mitosis. It contains a highly conserved N-terminal extension, which is essential in the regulation of APC/C E3 activity and the site of lysine residue to be modified on the substrate ${ }^{[10]}$. As an APC/C specific E2, UBE2C has a direct role in 
regulating the spindle assembly checkpoint by catalyzing multi-ubiquitination of one or more components of the APC-Cdc20-checkpoint protein complex ${ }^{[1]}$. Cells that overexpress UBE2C would fail to maintain spindle checkpoint activity after entering mitosis, resulting in chromosome missegregation and aneuploidy, which may eventually lead to tumor formation ${ }^{[11,12]}$.

Amplification and/or overexpression of UBE2C have been reported in many malignancies, and a high expression of UBE2C is associated with poor clinical outcomes. In this review, the pathological role of dysregulated UBE2C in gastrointestinal cancers and its potential role as a diagnostic and/or a prognostic marker as well as a therapeutic target in these cancers are discussed.

\section{Molecular biology of UBE2C and its role in cell cycle regulation}

UBE2C has eight homologs and contains 179 amino acids with the molecular weight of $19652 \mathrm{Da}^{[13]}$. Although small in size, it provides three to four binding sites to interact with other proteins simultaneously, including ubiquitin molecule, E1 enzyme, E3 enzyme, and the targeting substrate ${ }^{[14]}$. This is attributed to the unique spatial structure of the UBE2C protein that is featured with a four-stranded antiparallel $\beta$-sheet, a $3^{10}$-helix, and four $\alpha$-helices.

The ubiquitin-conjugating enzyme $2 \mathrm{C}$ (UBE2C) is essential for cell cycle progression as demonstrated by observations that mutation of the active site (position 114) inhibits the targeted destruction of mitotic cyclins. The influence of UBE2C on cell cycle progression is exerted during multiple phases of the cycle, during which the expression levels of the enzyme fluctuate. The major effect of UBE2C is to mediate the specific ubiquitination of ubiquitin ligase E3 enzyme known as anaphase-promoting complex/cyclosome (APC/C), that is involved in the completion of the cell cycle. Research has demonstrated that UBE2C promotes cell cycle progression from metaphase to anaphase through the regulation of mitotic spindle assessment checkpoints (SACs) and sister chromatid separation in an enzymatic cascade reaction ${ }^{[15]}$. During the mitotic $(\mathrm{M})$ phase, cell division cycle protein $20(\mathrm{Cdc} 20)$ is inhibited by SAC proteins, including Mad2, Bub3, and BubR 1 . When UBE2C is highly expressed, APC/C and UBE2C can degrade SAC by ubiquitination. The Cdc20 protein is dissociated by the SAC and forms APC/C-Cdc20 complexes ${ }^{[16,17]}$. The degradation of securin, a key substrate of $\mathrm{APC} / \mathrm{C}$, is enhanced by UBE2C through ubiquitination, releasing separase. Separase subsequently promotes the separation of sister chromatids and the onset of anaphase. Conversely, UBE2C can also inhibit the cell cycle via complexing with cyclin B, which activates Cdk1, a major inhibitory protein for cells to withdraw from mitosis and enter $\mathrm{S}$ phase (Figure 1). 


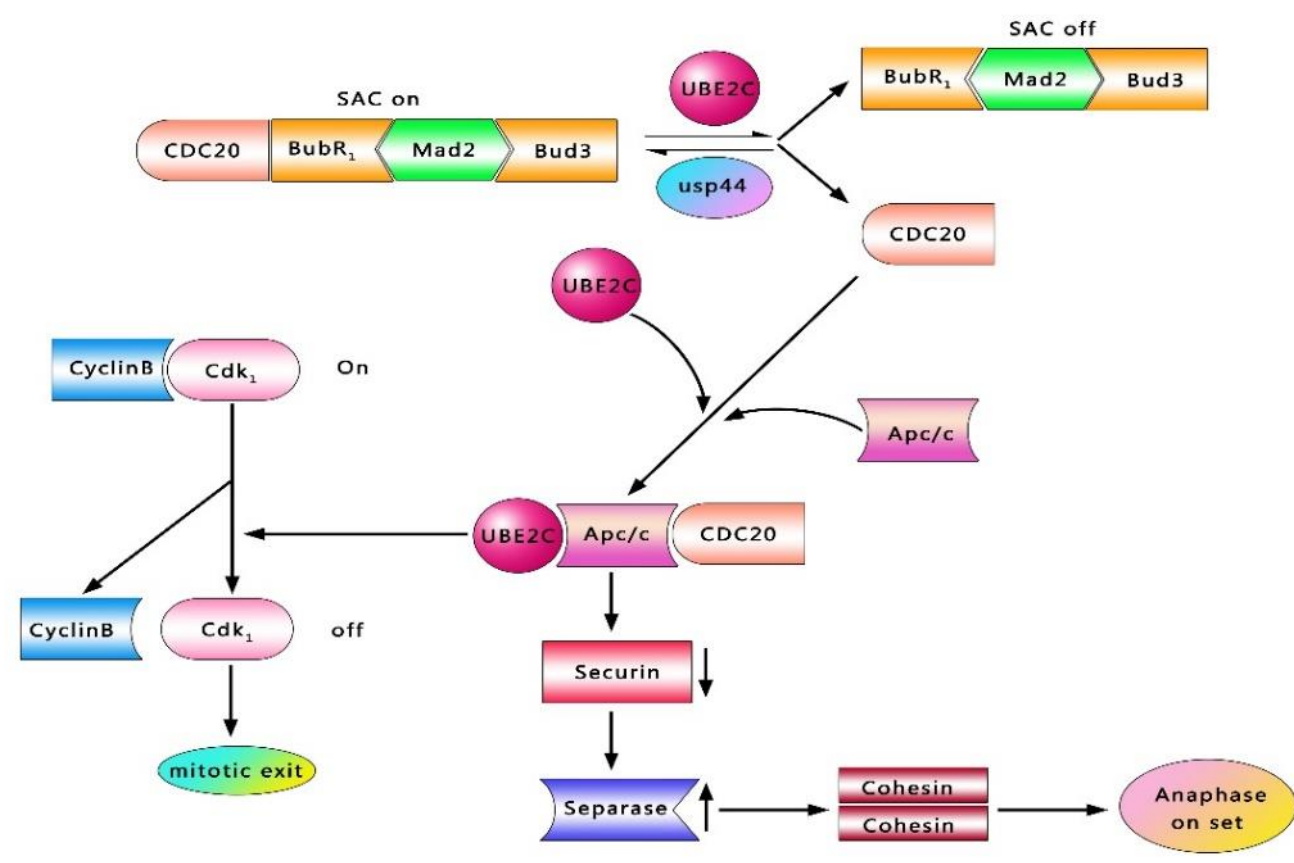

Figure 1. In M phase, Cdc20 is inhibited by SAC proteins, including Mad2, Bub3, and $\mathrm{BubR}_{1}$. APC/C and UBE2C can degrade SAC by ubiquitination, whereas USP44 antagonizes this process. The Cdc20 complexes with APC/C and UBE2C, thus enhancing the degradation of securin by ubiquitination, releasing separase. Separase promotes the separation of the sister chromatids and the onset of anaphase. The APC/C also degrades cyclin B resulting in the inhibition of Cdk1 and mitotic exit. SAC: spindle assembly checkpoint; APC/C: anaphase-promoting complex/cyclosome.

In 2003, Okamoto ${ }^{[18]}$ compared the expression of RNA in 25 normal human tissue samples with 24 tumor cell lines and tumor cell samples using reverse transcription polymerase chain reaction (RT-PCR). The results showed that 17 types of E2 genes were overexpressed among the samples and UBE2C protein was highly expressed in the majority of tumor cells but not in normal tissue samples. The expression of UBE2C can thus be considered as a tumor marker, and a high (relative) expression of the enzyme in cancer patients has been associated with a poor prognosis. A number of other studies have also implicated UBE2C in the pathogenesis of a range of different cancers. Pallante ${ }^{[19]}$ found that the levels of UBE2C protein in thyroid cancer cell line were 150 times those found in healthy cells; the transfection of NPA and TPC1 cell lines by SiRNA significantly reduced the growth of thyroid cancer cells. Furthermore, Rawat's breast cancer cell line experiments have shown that cell proliferation and cell adhesion-independent growth were significantly lower in a UBE2C-inhibited group than in a UBE2C-transfected cell line ${ }^{[20]}$. By observing a group of transgenic mice that express high levels of UBE2C, Van Ree ${ }^{[12]}$ established a model of UBE2C transgenic mice and found that this high expression of UBE2C could lead to the production of chromosome lag and aneuploid cells in mitosis. In a model of lung cancer induced by dimethylbenz $(\alpha)$ anthracene (DMBA), UBE2C mice were not only more likely to develop lung cancer, but also more serious forms of lung cancer.

The mitotic spindle checkpoint is inhibited by UBE2C and loses its monitoring functional capability. Uncontrolled activation of APC/C leads to chromosome misclassification and the production of aneuploidy cells. The expression of UBE2C is thus an important marker of chromosomal instability and is associated with malignant growth of tissues.

The UBE2C enzyme also plays a role in DNA damage. Cells expressing human papillomavirus type 16 (HPV-16) E6 and E7 proteins have the ability to enter as well as exit mitosis. It has been demonstrated that UBE2C plays an important role in this process by regulating Cdc20 and APC/C ${ }^{[21]}$. 


\section{Possible regulatory mechanisms and treatment of UBE2C}

Recent research has identified UBE2C as an important and complex regulatory factor in the ubiquitinproteasome system. Its role in the regulation of tumor growth has become a research hotspot, attracting significant attention across a range of different types of cancer due to its potential as a therapeutic target. The current scientific evidence in this area is summarized below.

In gastric cancer research, it has been shown that the inhibition of miR-17/20a expression in gastric cancer cells can cause the inhibition of cell proliferation. Results of quantitative real-time polymerase chain reaction (qRT-PCR) and western blot analysis have shown that UBE2C is a direct target of miR-17/20a, thus implicating UBE2C as a factor in the progression of gastric tumor growth. Further research has shown that the expression of UBE2C mRNA and protein is positively regulated by miR-17/20a ${ }^{[22]}$. A study conducted by Yang ${ }^{[23]}$ has suggested that gastric cancer cell lines can increase cisplatin-mediated apoptosis after UBE2C knockdown by siRNA. Other studies have shown that the inhibition of UBE2C by wild-type p53 is p21-E2F4-dependent, while the activation of UBE2C by mutant p53 is dependent on nuclear factor (nuclear factor $\mathrm{Y}, \mathrm{NF}-\mathrm{Y})^{[24]}$.

Colon cancer studies ${ }^{[25]}$ have shown that the inhibition of UBE2C reduces the growth rate of colon cancer cells (in-part through downregulation of cyclin B and ERK1) and increases the efficacy of chemotherapy drugs, such as irinotecan, $\mathrm{SN}-38$, and cetuximab (partially down-regulated by protein kinase B (AKT)). Furthermore, experiments on colorectal cancer cells conducted by Prashant confirmed that the protease-inhibitor drug, bortezomib, mediates the increased expression of cyclin A and cyclin B by downregulating UBE2C; bortezomib-treated nude mice exhibited a significant increase in cyclin A and cyclin B expression compared to untreated mice, and downregulation of UBE2C increased the sensitivity of colon cancer cells to apoptosis mediated by bortezomib and the chemotherapeutic drug, oxaliplatin ${ }^{[26]}$. Cyclin A and cyclin B play important roles in the regulation of cell mitosis. The same research further showed that bortezomib can stabilize cyclin A and cyclin B expression at both translation and transcriptional levels.

Another colorectal cancer study ${ }^{[27]}$ suggested that a high expression of UBE2C protein in tumor cells is correlated with tumor cell resistance to apoptosis, which is mediated by $\mathrm{N}$-acetyl-leu-leu-norleucinal (ALLN), and that the inhibition of UBE2C is likely to lead to cell apoptosis. The suggested mechanism for this effect is that the low level of UBE2C increases the activity of caspase 8 and caspase 3 in the presence of ALLN, thus impairing the survival of tumor cells.

In another research ${ }^{[28]}$, it has been shown that the inhibition of UBE2C expression can increase the sensitivity to both radiation therapy and chemotherapy. In addition, small molecule inhibitors for UBE2C are being studied in this field using docking studies.

Studies of other cancer types have shown that the knockdown of UBE2C reduces osteosarcoma proliferation and invasion by inhibiting Ki67, matrix metalloproteinase-3, and matrix metalloproteinase-9 ${ }^{[29]}$. In lung cancer studies ${ }^{[30]}$, UBE2C was found to be negatively correlated with mutant p53 and epidermal growth factor receptor (EGFR), which are involved in tumorigenesis. Further, in a study of castrationresistant prostate cancer (CRPC) cell lines - abl and C4-2B, it has been found that a cell cycle inhibitor, CCI-779, inhibited the expression of UBE2C mRNA and protein as well as interfered with cell growth.

\section{UBE2C and gastrointestinal cancers}

In recent years, research on the involvement of UBE2C in gastrointestinal cancers have confirmed that it is highly expressed in esophageal, colon, gastric, liver, and pancreatic cancers. Furthermore, correlation studies have suggested that UBE2C expression is associated with the development of tumor cells. These studies are reviewed below. 
Lin ${ }^{[31]}$ found that the transfection of Seg-1 cell line with plasmids that failed to express UBE2C resulted in a reduction in the proliferation of esophageal cancer cells and the suspension of these cells in the mitotic phase. After the transfection of cells with RNAi, the cell cycle was disturbed, cell proliferation was inhibited and the number of G2 phase cells peaked after 72 hours. The cell proliferation of Seg-1 cell line reduced after treating with protease inhibitor.

Fujita ${ }^{[32]}$ found that UBE2C is not usually expressed in normal colonic tissues but markedly expressed in colon cancer tissues. In that study, the authors reported that there was an overexpression of UBE2C in the DLD1 cell line and the culture doubling time of tumor cells in the overexpressed group was markedly lower compared to the control group. In addition, similar results have been observed in agarose growth experiments. The opposite effect was noted in colon cancer cell lines where the UBE2C gene had been knocked out. The study found that there is an association between the number of mitotic cells and the level UBE2C expression. A separate study by Chen ${ }^{[33]}$ similarly found that the expression of UBE2C in normal colon cells in vitro is essentially negative. The authors studied cell proliferation and invasion in a HT-29 cell line transfected with UBE2C plasmid (HT-29/UbcH10). The results showed that UBE2C overexpression promoted the proliferation and invasion of tumor cells compared to the control group. However, UBE2C expression was significantly lower in HT-29/UbcH10-RNAi cells that had been transfected with siRNA to block UBE2C. The same study performed a basement membrane invasion test, and the authors reported a significant difference in the number of passing through the basement membrane between the transfected group and the control group. Collectively, these findings indicate that UBE2C expression increases the generation of colon cancer cells and accelerates tumor progression.

A number of clinical studies have shown that UBE2C protein expression is raised in colon cancer tissues ${ }^{[23-25]}$. Yang confirmed that the expression of UBE2C is raised in gastric cancer cell lines compared to normal gastric tissues and observed that the proliferation of gastric cancer cells decreased following a knockdown of UBE2C expression. Immunohistochemistry analysis further revealed that UBE2C protein is highly expressed in the majority of gastric cancer tissue samples, whilst its expression levels are low in adjacent interstitial tissues ${ }^{[23]}$. Okamoto ${ }^{[18]}$ used qRT-PCR to measure UBE2C mRNA expression and reported it to be high in gastric cancer tissues but low or absent in normal tissues. Furthermore, it was found that the overexpression of UBE2C in NIH3T3 cells, that had been transfected with appropriate expression plasmids, increased the binding of bromodeoxyuridine, promoted cell proliferation, and accelerated colony formation in the agarose assay. The study concluded that UBE2C promotes gastric cancer cell proliferation and the transformation of normal cells into malignant cells.

In a study about liver cancer ${ }^{[34]}$, the expression levels of UBE2C mRNA as measured by qRT-PCR were reported to be significantly higher in a range of cancer cell lines compared to normal liver tissue. The expression of UBE2C mRNA in hepatocarcinoma cell line was found to be significantly higher than that in a normal liver cell line and the difference was significant.

Pancreatic ductal adenocarcinoma (PDA) accounts for more than $90 \%$ of pancreatic cancers. A research on UBE2C expression in 94 PDA tumor tissues and adjacent normal pancreatic tissues showed that the expression of UBE2C protein in ductal carcinoma tissues was higher than that in paracancerous tissue $^{[35]}$.

\section{UBE2C and clinicopathological characteristics of gastrointestinal cancers}

Evidence from available studies indicates that UBE2C expression may be a potential prognostic marker or therapeutic target in gastrointestinal cancers. Clinical studies have shown that UBE2C expression is correlated with a variety of clinical and pathological parameters as well as the prognosis in patients with gastrointestinal tumors. These studies are summarized below.

In a study about esophageal cancer ${ }^{[36]}$, significant differences in lymphatic invasion, lymph node 
metastasis, and TNM staging were reported between patients with high UBE2C expression and those with low expression of the protein. The 50\% survival rate of these two groups also differed significantly.

Chen ${ }^{[33]}$ measured the expression of UBE2C in 45 cases of colorectal cancer and in corresponding adjacent normal tissue samples. The study reported that the expression was 10 times higher in the former compared with the latter. There were no statistically significant differences in UBE2C protein expression with age, sex, or tumor size among the samples, but there was an association with lymph node metastasis and pathological differentiation. Studies by Fujita ${ }^{[32]}$ and Cacciola ${ }^{[25]}$ have confirmed these findings and further identified an association between UBE2C expression and mutations in KRAS oncogene. Studies have also identified that UBE2C expression and cancer cell proliferation in colon cancer tissues are lower in older patients than in younger ones; in addition, distant metastases is rare in older patients ${ }^{[26]}$. This suggests that UBE2C expression is associated with the proliferation of tumors at adjacent tissue sites.

In 2013, Zhao [35] reported that UBE2C protein expression is related to pancreatic ductal adenocarcinoma differentiation, clinical stage, and lymph node metastasis. Zhao hypothesized that UBE2C plays an important role in the progression of pancreatic cancer and proposed that it could be used as a prognostic indicator.

In 2011, a study ${ }^{[34]}$ about liver cancer investigated the associations between UBE2C mRNA expression and various factors, such as age, sex, tumor size, tumor size, portal vein invasion, histopathological differentiation, TNM staging, envelope formation, alpha-fetoprotein, hepatitis B surface antigen (HBsAg), and other clinical pathological parameters. The expression of UBE2C mRNA was found to be significantly correlated to histopathological differentiation, tumor size, TNM staging, and portal vein invasion. The authors concluded that the overexpression of UBE2C in hepatocellular carcinoma may be indicative of a poor prognosis and is therefore of clinical significance.

In gastric cancer, the expression of UBE2C was found to have correlation with lymphatic metastasis, serosa invasion, TNM staging, and Lauren's classification. Univariate analysis showed that the overexpression of UBE2C has associations with poor prognosis. The multivariate analysis demonstrated that the expression of UBE2C, lymphatic metastasis, and TNM staging are independent prognostic indicators ${ }^{[37]}$.

\section{Conclusion and perspectives}

The collective body of currently available evidence suggests that UBE2C plays an important role in the initiation, development, as well as proliferation of gastrointestinal tumors and may represent a novel therapeutic target and/or prognostic marker for gastrointestinal cancers.

Table 1. Studies of UBE2C expression in human gastrointestinal cancers

\begin{tabular}{ll}
\hline Type of gastrointestinal cancer & \multicolumn{1}{c}{ Findings } \\
\hline $\begin{array}{l}\text { Esophageal cancer } \\
\text { Colon cancer }\end{array}$ & $\begin{array}{l}\text { After transfection of RNAi, cell proliferation is inhibited. } \\
\text { High expression levels in colon cancer tissues. UBE2C overexpression } \\
\text { promotes the proliferation and invasion of the tumor. } \\
\text { Gastric cancer }\end{array}$ \\
& $\begin{array}{l}\text { Gastric cancer cell lines have higher expression of UBE2C compared with } \\
\text { normal gastric tissues. Gastric cancer cell proliferation decreases after }\end{array}$ \\
& UBE2C expression has been knocked down. \\
Eiver cancer & $\begin{array}{l}\text { Expression of UBE2C mRNA in adjacent normal tissues is much lower } \\
\text { than that in hepatocellular carcinoma tissues. } \\
\text { Eancreatic ductal }\end{array}$ \\
Expression of UBE2C protein in ductal carcinoma tissues is higher than \\
that in para-cancerous tissues.
\end{tabular}


Table 2. UBE2C and clinicopathological characteristics of gastrointestinal cancers

\begin{tabular}{|c|c|c|}
\hline Type of gastrointestinal cancer & Findings & References \\
\hline Esophageal cancer & $\begin{array}{l}\text { Its expression correlates with lymphatic invasion, lymph node } \\
\text { metastasis, and TNM staging. Overexpression of UBE2C is associated } \\
\text { with poor prognosis. }\end{array}$ & {$[36]$} \\
\hline Colon cancer & $\begin{array}{l}\text { Its expression is associated with lymph node metastasis and pathological } \\
\text { differentiation. Its expression is lower in elderly patients compared to } \\
\text { younger patients. }\end{array}$ & {$[25,32,33]$} \\
\hline Liver cancer & $\begin{array}{l}\text { Its expression significantly correlates with histopathological } \\
\text { differentiation, tumor size, TNM staging, and portal vein invasion. }\end{array}$ & {$[34]$} \\
\hline $\begin{array}{l}\text { Pancreatic ductal } \\
\text { adenocarcinoma }\end{array}$ & $\begin{array}{l}\text { Its expression is related to pancreatic ductal adenocarcinoma } \\
\text { differentiation, clinical stage, and lymph node metastasis. UBE2C can be } \\
\text { used as an independent predictor of prognosis. }\end{array}$ & {$[35]$} \\
\hline Gastric cancer & $\begin{array}{l}\text { Its expression correlates with lymphatic metastasis, serosa invasion, } \\
\text { TNM staging, and Lauren's classification. UBE2C can be used as an } \\
\text { independent predictor of prognosis. }\end{array}$ & {$[37]$} \\
\hline
\end{tabular}

\section{Funding}

This research is supported by the NSFC grant (81773178) and belongs to stage result of Hebei Provincial Health and Family Planning Commission Project (Key Science and Technology Research Program) with grant number: 20171069.

\section{Disclosure statement}

The authors declare that there is no conflict of interest.

\section{References}

[1] Ohtake F, Tsuchiya H, 2016, The Emerging Complexity of Ubiquitin Architecture. Journal of Biochemistry, 161(2): 125-133.

[2] Yau R, Rape M, 2016, The Increasing Complexity of the Ubiquitin Code. Nature Cell Biology, 18(6): 579-586.

[3] Handley PM, Mueckler M, Siegel NR, et al., 1991, Molecular Cloning, Sequence, and Tissue Distribution of the Human Ubiquitin-Activating Enzyme E1. Proceedings of the National Academy of Sciences of the United States of America, 88(1): 258-262.

[4] Jin J, Li X, Gygi SP, et al., 2007, Dual E1 Activation Systems for Ubiquitin Differentially Regulate E2 Enzyme Charging. Nature, 447(7148): 1135-1138.

[5] Michelle C, Vourc'h P, Mignon L, et al., 2009, What Was the Set of Ubiquitin and Ubiquitin-Like Conjugating Enzymes in the Eukaryote Common Ancestor? Journal of Molecular Evolution, 68(6): 616-628.

[6] Li W, Bengtson MH, Ulbrich A, et al., 2008, Genome-Wide and Functional Annotation of Human E3 Ubiquitin Ligases Identifies MULAN, a Mitochondrial E3 that Regulates the Organelle's Dynamics and Signaling. Plos One, 3(1): e1487. 
[7] Burroughs AM, Jaffee M, Iyer LM, et al., 2008, Anatomy of the E2 Ligase Fold: Implications for Enzymology and Evolution of Ubiquitin/Ub-Like Protein Conjugation. Journal of Structural Biology, 162(2): 205-218.

[8] Van Wijk SJ, Timmers HT, 2010, The Family of Ubiquitin-Conjugating Enzymes (E2s): Deciding Between Life and Death of Proteins. The FASEB Journal, 24(4): 981-993.

[9] Winn PJ, Religa TL, Battey JND, et al., 2004, Determinants of Functionality in the Ubiquitin Conjugating Enzyme Family. Structure (Cambridge), 12(9): 1563-1574.

[10] Summers MK, Pan B, Mukhyala K, et al., 2008, The unique N terminus of the UbcH10 E2 enzyme controls the threshold for APC activation and enhances checkpoint regulation of the APC. Molecular Cell, 31(4): 544-556.

[11] Reddy SK, Rape M, Margansky WA, et al., 2007, Ubiquitination by the Anaphase-Promoting Complex Drives Spindle Checkpoint Inactivation. Nature, 446(7138): 921-925.

[12] Van Ree JH, Jeganathan KB, Malureanu L, et al., 2010, Overexpression of the E2 UbiquitinConjugating Enzyme UbcH10 Causes Chromosome Missegregation and Tumor Formation. The Journal of Cell Biology, 188(1): 83-100.

[13] Townsley FM, Aristarkhov A, Beck S, et al., 1997, Dominant-Negative Cyclin-Selective Ubiquitin Carrier Protein E2-C/UbcH10 Blocks Cells in Metaphase. Proceedings of the National Academy of Sciences of the United States of America, 94(6): 2362-2367.

[14] Jiang F, Basavappa R, 1999, Crystal Structure of the Cyclin-Specific Ubiquitin-Conjugating Enzyme from Clam, E2-C, at 2.0 A Resolution. Biochemistry, 38(38): 6471-6478.

[15] Yanagida M, 2005, Basic Mechanism of Eukaryotic Chromosome Segregation. Philosophical Transactions Biological Sciences, 360(1455): 609-621.

[16] Williamson A, Banerjee S, Zhu X, et al., 2011, Regulation of Ubiquitin Chain Initiation to Control the Timing of Substrate Degradation. Molecular Cell, 42(6): 744-757.

[17] Hao Z, Zhang H, Cowell J, 2012, Ubiquitin-Conjugating Enzyme UBE2C: Molecular Biology, Role in Tumorigenesis, and Potential as a Biomarker. Tumor Biology, 33(3): 723-730.

[18] Okamoto Y, Ozaki T, Miyazaki K, et al., 2003, UbcH10 is the Cancer-Related E2 UbiquitinConjugating Enzyme. Cancer Research, 63(14): 4167-4173.

[19] Pallante P, Berlingieri MT, Troncone G, et al., 2005, UbcH10 Overexpression May Represent a Marker of Anaplastic Thyroid Carcinomas. British Journal of Cancer, 93(4): 464.

[20] Rawat A, Gopal G, Selvaluxmy G, et al., 2013, Inhibition of Ubiquitin Conjugating Enzyme UBE2C Reduces Proliferation and Sensitizes Breast Cancer Cells to Radiation, Doxorubicin, Tamoxifen and Letrozole. Cellular oncology, 36(6): 459-467.

[21] Patel D, McCance DJ, 2010, Compromised Spindle Assembly Checkpoint Due to Altered Expression of UbcH10 and Cdc20 in Human Papillomavirus Type 16 E6- and E7-Expressing Keratinocytes. Journal of Virology, 84(21): 10956-10964.

[22] Cheng J, Kang X, Zhang S, et al., 2007, SUMO-Specific Protease 1 is Essential for Stabilization of HIF1 $\alpha$ during Hypoxia. Cell, 131(3): 584-595.

[23] Yang M, Qu Y, Shi R, et al., 2016, Ubiquitin-Conjugating Enzyme Ubch10 Promotes Gastric Cancer Growth and is a Potential Biomarker for Gastric Cancer. Oncology Reports, 36(2): 779-786.

[24] Bajaj S, Alam SK, Roy KS, et al., 2016, E2-Ubiquitin Conjugating Enzyme, UBE2C, is Reciprocally Regulated by Wild-Type and Gain-Of-Function Mutant p53. Journal of Biological Chemistry, 291(27): 14231-14247. 
[25] Cacciola NA, Calabrese C, Malapelle U, et al., 2016, UbcH10 Expression Can Predict Prognosis and Sensitivity to the Antineoplastic Treatment for Colorectal Cancer Patients. Molecular Carcinogenesis, 55(5): 793-807.

[26] Bavi P, Uddin S, Ahmed M, et al., 2011, Bortezomib Stabilizes Mitotic Cyclins and Prevents Cell Cycle Progression Via Inhibition of UBE2C in Colorectal Carcinoma. The American Journal of Pathology, 178(5): 2109-2120.

[27] Li SZ, Song Y, Zhang HH, et al., 2014, UbcH10 Overexpression Increases Carcinogenesis and Blocks ALLN Susceptibility in Colorectal Cancer. Scientific Reports, 4: 6910.

[28] Sabitha K, Rajkumar T, 2012, Identification of Small Molecule Inhibitors Against UBE2C by Using Docking Studies. Bioinformation, 8(21): 1047.

[29] Wang ST, Li DZ, Li JM, et al., 2015, Lentivirus-Mediated RNA Interference Targeting UbcH10 Reduces Cell Growth and Invasion of Human Osteosarcoma Cells Via Inhibition of Ki-67 and Matrix Metalloproteinases. Oncology Letters, 9(5): 2171-2176.

[30] Pallante P, Malapelle U, Berlingieri MT, et al., 2013, UbcH10 Overexpression in Human Lung Carcinomas and Its Correlation with EGFR and p53 Mutational Status. European Journal of Cancer, 49(5): 1117-1126.

[31] Lin J, Raoof DA, Wang Z, et al., 2006, Expression and Effect of Inhibition of the UbiquitinConjugating Enzyme E2C on Esophageal Adenocarcinoma. Neoplasia, 8(12): 1062-1071.

[32] Fujita T, Ikeda H, Taira N, et al., 2009, Overexpression of UbcH10 Alternates the Cell Cycle Profile and Accelerate the Tumor Proliferation in Colon Cancer. BMC Cancer, 9(1): 87.

[33] Chen S, Chen Y, Hu C, et al., 2010, Association of Clinicopathological Features with UbcH10 Expression in Colorectal Cancer. Journal of Cancer Research and Clinical Oncology, 136(3): 419-426.

[34] Han SS, Liu QG, Yao YM, et al., 2011, UbcH10 Expression in Hepatocellular Carcinoma and Its Clinicopathological Significance. Nan Fang Yi Ke Da Xue Xue Bao (Journal of Southern Medical University), 31(2): 280-284.

[35] Zhao ZK, Wu WG, Chen L, et al., 2013, Expression of UbcH10 in Pancreatic Ductal Adenocarcinoma and Its Correlation with Prognosis. Tumor Biology, 34(3): 1473-1477.

[36] Matsumoto A, Ishibashi Y, Urashima M, et al., 2014, High UbcH10 Protein Expression as a Marker of Poor Prognosis in Esophageal Squamous Cell Carcinoma. Anticancer Research, 34(2): 955-961.

[37] Zhang HQ, Zhao G, Ke B, et al., 2018, Overexpression of UBE2C Correlates with Poor Prognosis in Gastric Cancer Patients. European Review for Medical and Pharmacological Sciences, 22(6): 16651671. 